\title{
Primary and Secondary Dissociation from Allyl Iodide Excited at 193 nm: Centrifugal Effects in the Unimolecular Dissociation of the Allyl Radical ${ }^{\dagger}$
}

\author{
D. E. Szpunar, M. L. Morton, $;$ and L. J. Butler* \\ The James Franck Institute and Department of Chemistry, The University of Chicago, Chicago, Illinois 60637 \\ P. M. Regan ${ }^{\S}$ \\ Chemical Sciences Division, Lawrence Berkeley National Laboratory, Berkeley, California 94720
}

Received: February 11, 2002; In Final Form: May 15, 2002

\begin{abstract}
In the work presented here, we used photofragment translational spectroscopy and $\mathrm{H}$ atom Rydberg timeof-flight (HRTOF) spectroscopy to study the primary photofragmentation channels of allyl iodide excited at $193 \mathrm{~nm}$ and the ensuing dissociation of the nascent allyl radicals as a function of their internal energy. Two $\mathrm{C}-\mathrm{I}$ bond fission channels were found to produce the allyl radical, one channel forming $\left.\mathrm{I}^{2} \mathrm{P}_{3 / 2}\right)$ and the other forming $\mathrm{I}\left({ }^{2} \mathrm{P}_{1 / 2}\right)$. The nascent allyl radicals are dispersed as a function of the translational energy imparted from the photolysis and therefore by their internal energy. Although all of the $\mathrm{I}\left({ }^{2} \mathrm{P}_{3 / 2}\right)$ and a portion of the $\mathrm{I}\left({ }^{2} \mathrm{P}_{1 / 2}\right)$ channel allyl radical products have enough internal energy to overcome the $60 \mathrm{kcal} / \mathrm{mol}$ barrier to form allene $+\mathrm{H}$, the data showed that a substantial fraction of the allyl radicals from the $\mathrm{I}\left({ }^{2} \mathrm{P}_{1 / 2}\right)$ channel that formed with internal energies as high as $15 \mathrm{kcal} / \mathrm{mol}$ above the $60 \mathrm{kcal} / \mathrm{mol}$ barrier were stable to $\mathrm{H}$ atom loss. The stability is due to centrifugal effects caused by significant rotational energy imparted to the allyl radical during photolysis and the small impact parameter and reduced mass characterizing the loss of an $\mathrm{H}$ atom from an allyl radical to form allene $+\mathrm{H}$. A photoionization efficiency (PIE) curve identified the major $\mathrm{C}_{3} \mathrm{H}_{4}$ secondary dissociation products as allene. Comparison of the mass 40 signal in the TOF spectra at two photoionization energies showed that branching to $\mathrm{H}+$ propyne does not occur at near-threshold internal energies, indicating that the experimentally determined allyl $\rightarrow 2$-propenyl radical isomerization barrier, which is lower than recent ab initio calculations of the barrier by $\sim 15 \mathrm{kcal} / \mathrm{mol}$, is far too low.
\end{abstract}

\section{Introduction}

This paper presents a study of the photodissociation of allyl iodide at $193 \mathrm{~nm}$ as well as the secondary dissociation of the nascent allyl radicals. The study is unique in that it disperses the nascent allyl radicals by the velocity imparted in the primary photolysis step, thus, in effect, dispersing the allyl radical by internal energy. This allows us to determine what unimolecular dissociation products are formed from the allyl radical as a function of its internal energy and to probe the marked effect of the centrifugal barrier on the unimolecular dissociation of the allyl radical.

We first review previous work on the photochemistry of allyl iodide. Despite its role as a precursor for the allyl radical, ${ }^{1-11}$ little is known about the photochemistry of allyl iodide. There are two UV spectra in the literature, both of which display a strong, broad, structureless absorption band peaking near 200 $\mathrm{nm}$. This band was assigned to an $\mathrm{n}_{\mathrm{I}} \rightarrow \pi^{*} \mathrm{C}_{\mathrm{C}} \mathrm{C}$ transition by Worrell, ${ }^{12}$ whereas Boschi and Salahub ${ }^{13}$ did not give an assignment. Using low-level ab initio calculations, Parsons et al. ${ }^{14}$ characterized the transition as having primarily $\pi \rightarrow$ Rydberg and $\pi_{\mathrm{C}=\mathrm{C}} / \mathrm{n}_{\mathrm{I}} \rightarrow \sigma^{*}{ }_{\mathrm{C}-\mathrm{I}} / \pi^{*}{ }_{\mathrm{C}=\mathrm{C}}$ character. Emission spectroscopy of allyl iodide excited at $199.7 \mathrm{~nm}$ helped to

\footnotetext{
† Part of the special issue "John C. Tully Festschrift".

* Corresponding author. E-mail: ljb4@midway.uchicago.edu.

$\doteqdot$ Current address: Chemistry Division, Argonne National Laboratory, Argonne, Illinois 60439.

$\$$ Current address: Archbishop Ilsley School, Victoria Road, Ancocks Green, Birmingham, U.K.
}

characterize the Franck-Condon region of the excited state. ${ }^{14}$ A strong $\mathrm{C}=\mathrm{C}$ stretch provided support for a $\pi \rightarrow$ Rydberg contribution because the loss of a $\pi$ electron would increase the $\mathrm{C}=\mathrm{C}$ bond length. The lack of a $\mathrm{C}-\mathrm{I}$ stretch gave no evidence of a $\pi_{\mathrm{C}=\mathrm{C}} / \mathrm{n}_{\mathrm{I}} \rightarrow \sigma^{*}{ }_{\mathrm{C}-\mathrm{I}} / \pi^{*}{ }_{\mathrm{C}=\mathrm{C}}$ contribution during the initial nuclear dynamics of the excited state. The emission spectroscopy of allyl iodide excited at 224.63, 237.11, 237.29, ${ }^{10}$ and $240.51 \mathrm{~nm}^{11}$ has also been presented in the literature.

Prior work on the gas-phase photolysis of allyl iodide at 355 $n m$ shows iodine as a product in both its ground state, $\mathrm{I}\left({ }^{2} \mathrm{P}_{3 / 2}\right)$, and in the excited spin-orbit state, $\mathrm{I}\left({ }^{2} \mathrm{P}_{1 / 2}\right) .{ }^{15}$ In the same study, vibrational overtone excitation of $v_{5}$ (methylene symmetric stretch) was seen to produce $\mathrm{I}\left({ }^{2} \mathrm{P}_{3 / 2}\right)$. In another study, the rate constant for the unimolecular dissociation of allyl iodide through $v_{6}$ (methylene asymmetric stretch) overtone excitation to produce $\mathrm{I}\left({ }^{2} \mathrm{P}_{3 / 2}\right)$ was determined. ${ }^{16}$ Formation of $\mathrm{I}\left({ }^{2} \mathrm{P}_{1 / 2}\right)$ has been observed following excitation of allyl iodide at $266 \mathrm{~nm} .{ }^{17}$ The lifetime of allyl iodide excited at $159 \mathrm{~nm}$ has also been investigated using a pump-probe REMPI technique. ${ }^{18}$ In other work, allyl iodide photolyzed using a $\mathrm{Hg}$ arc lamp in an $\mathrm{Ar}$ matrix was found to produce primarily allene and $\mathrm{HI} .{ }^{19} \mathrm{~A} \mathrm{Hg}$ lamp was also used to photolyze allyl iodide in aromatic solvents to study the formation of allyl arenes. ${ }^{20}$

The experiments detailed here first utilize photofragment translational spectroscopy to classify the product channels of allyl iodide photolyzed at $193 \mathrm{~nm}$. Reactions $1-5$ are some of the possible reaction channels of allyl iodide upon excitation at 193 nm..$^{21,22}$ 


$$
\begin{array}{lrl}
\mathrm{C}_{3} \mathrm{H}_{5} \mathrm{I} \rightarrow \mathrm{C}_{3} \mathrm{H}_{5}+\mathrm{I}\left({ }^{2} \mathrm{P}_{3 / 2}\right) & \Delta H^{\circ}{ }_{298 \mathrm{~K}}=42.6 \mathrm{kcal} / \mathrm{mol} \\
\left.\mathrm{C}_{3} \mathrm{H}_{5} \mathrm{I} \rightarrow \mathrm{C}_{3} \mathrm{H}_{5}+\mathrm{I}^{2}{ }^{2} \mathrm{P}_{1 / 2}\right) & \Delta H^{\circ}{ }_{298 \mathrm{~K}}=64.3 \mathrm{kcal} / \mathrm{mol} \\
\mathrm{C}_{3} \mathrm{H}_{5} \mathrm{I} \rightarrow \mathrm{CH}_{2}=\mathrm{C}=\mathrm{CH}_{2}+\mathrm{HI} & \Delta H^{\circ}{ }_{298 \mathrm{~K}}=28.0 \mathrm{kcal} / \mathrm{mol} \\
& & \Delta H^{\circ}{ }_{298 \mathrm{~K}}=26.8 \mathrm{kcal} / \mathrm{mol} \\
\mathrm{C}_{3} \mathrm{H}_{5} \mathrm{I} \rightarrow \mathrm{CH} \equiv \mathrm{CCH}_{3}+\mathrm{HI} & \Delta H^{\circ}{ }_{298 \mathrm{~K}}=33.8 \mathrm{kcal} / \mathrm{mol}
\end{array}
$$

Following the initial photodissociation, the energy available for the relative translational energy and internal energy of the products, $E_{\text {avl }}$, is given by

$$
E_{\text {avl }}=h v-\Delta H_{0 \mathrm{~K}}^{\circ}+E_{\text {initial }}=E_{\text {int radical }}+E_{\mathrm{T}}
$$

where $E_{\text {initial }}$, the initial internal energy of the parent $\mathrm{C}_{3} \mathrm{H}_{5} \mathrm{I}$ molecule, is neglected in this paper because it is small compared to the other uncertainties present. The $\Delta H^{\circ}$ values include the energy required to form the relevant spin-orbit state of the I atom, and in all calculations of $E_{\text {int }}$ to follow in this paper, $\Delta H^{\circ}{ }_{0 \mathrm{~K}}$ was approximated by $\Delta H^{\circ}{ }_{298} \mathrm{~K}$. With these approximations, reactions 1 and 2 have $E_{\text {avl }}$ values of 105.3 and $83.6 \mathrm{kcal} /$ mol, respectively. Depending on how much of the $E_{\text {avl }}$ is partitioned to the kinetic energy of the I and allyl radical fragments, the allyl radical may be left with enough internal energy to undergo several possible secondary reactions, a few of which are shown in eqs 7-9 with $\Delta H^{\circ}{ }_{0 \mathrm{~K}}$ and barriers listed for each channel calculated by Davis et al. ${ }^{23}$ For the reactions with 2-propenyl or 1-propenyl radicals as the reactant, we show Davis et al.'s ab initio energetic barrier to each channel (corrected for zero-point energy) with respect to the zero-point level of the 2-propenyl or 1-propenyl radical, respectively, and give in parentheses the same barrier height with respect to the zero-point level of the allyl radical.

$$
\begin{aligned}
& \mathrm{C}_{3} \mathrm{H}_{5} \text { (allyl) } \rightarrow \mathrm{CH}_{2}=\mathrm{C}=\mathrm{CH}_{2} \text { (allene) }+\mathrm{H} \\
& \Delta H_{0 \mathrm{~K}}^{\circ}=54.4 \mathrm{kcal} / \mathrm{mol} \quad 60.0 \mathrm{kcal} / \mathrm{mol} \text { barrier } \\
& \mathrm{C}_{3} \mathrm{H}_{5} \text { (allyl) } \rightarrow \mathrm{CH}_{2}=\mathrm{CCH}_{3} \text { (2-propenyl) } \\
& \Delta H^{\circ}{ }_{0 \mathrm{~K}}=19.4 \mathrm{kcal} / \mathrm{mol} \quad 63.8 \mathrm{kcal} / \mathrm{mol} \text { barrier } \\
& \mathrm{CH}_{2}=\mathrm{CCH}_{3} \text { (2-propenyl) } \rightarrow \mathrm{CH}_{2}=\mathrm{C}=\mathrm{CH}_{2} \text { (allene) }+\mathrm{H} \\
& \mathrm{CH}_{2}=\mathrm{CCH}_{3} \text { (2-propenyl) } \rightarrow \mathrm{CH} \equiv \mathrm{CCH}_{3} \text { (propyne) }+\mathrm{H} \\
& \mathrm{C}_{3} \mathrm{H}_{5} \text { (allyl) } \rightarrow \mathrm{CH}=\mathrm{CHCH}_{3} \text { (1-propenyl) } \\
& \Delta H^{\circ}{ }_{0 \mathrm{~K}}=23.0 \mathrm{kcal} / \mathrm{mol} \quad 64.0 \mathrm{kcal} / \mathrm{mol} \text { barrier } \\
& \mathrm{CH}=\mathrm{CHCH}_{3} \text { (1-propenyl) } \rightarrow \mathrm{HC} \equiv \mathrm{CH}+\mathrm{CH}_{3} \\
& \Delta H^{\circ}{ }_{0 \mathrm{~K}}=23.2 \mathrm{kcal} / \mathrm{mol} \quad 33.0(56.0) \mathrm{kcal} / \mathrm{mol} \text { barrier }
\end{aligned}
$$

Reactions $8^{\prime}$ and $8^{\prime \prime}$ are dissociative reactions following isomerization to the 2-propenyl radical, which branches to $\mathrm{H}+$ propyne as the major product channel whereas the allyl radical gives only allene $+\mathrm{H}$ directly. Therefore, detecting branching to the propyne $+\mathrm{H}$ channel indicates that the allyl $\rightarrow$ 2-propenyl radical isomerization is competitive with the direct $\mathrm{C}-\mathrm{H}$ bond fission from the allyl radical to produce allene $+\mathrm{H}$. The experimentally determined allyl $\rightarrow 2$-propenyl radical isomerization barrier ${ }^{24}$ is $15 \mathrm{kcal} / \mathrm{mol}$ lower than the calculated values, so these experiments can definitively resolve which is correct.

Whereas this work allows us to study the unimolecular dynamics of the allyl radical dispersed by internal energy in the ground electronic state, the results may be compared to prior photodissociation studies of the allyl radical because the photolysis of the allyl radical is believed to proceed via internal conversion to the ground electronic state. One important difference, however, is that our nascent hot allyl radicals have considerable energy in both vibrational and rotational degrees of freedom, whereas UV-excited allyl radicals formed in a jet have a much cooler rotational temperature. Several studies have detailed the dynamics of vibrationally hot allyl radicals formed via UV excitation of ground-state allyl radicals. ${ }^{3-5,7 a, 25}$ Stranges et al. ${ }^{4}$ used photofragment translational spectroscopy to study the photodissocation dynamics of the allyl radical produced through the pyrolysis of allyl iodide. Photolysis at 248 and 351 $n m$ excited the allyl radical to the $\tilde{\mathrm{C}}\left(2^{2} \mathrm{~B}_{1}\right)$ and $\tilde{\mathrm{A}}\left(1^{2} \mathrm{~B}_{1}\right)$ states, which then relaxed to the ground electronic state through internal conversion. At $248 \mathrm{~nm}$, the hot allyl radicals underwent $\mathrm{C}-\mathrm{H}$ fission $(84 \%)$ or produced methyl + acetylene (reaction $9,16 \%$ ). It is thought that some of the $\mathrm{H}$ loss may be due to predissociation through a higher excited state. RRKM calculations using the experimentally determined $48.6 \mathrm{kcal} / \mathrm{mol}$ allyl $\rightarrow$ 2-propenyl radical isomerization barrier ${ }^{24}$ predicted that $50 \%$ of the $\mathrm{H}$ loss products should result from the direct loss of $\mathrm{H}$ to produce allene (reaction 7) and that $50 \%$ should produce either allene or propyne $+\mathrm{H}$ following isomerization to 1- and 2-propenyl radicals (reactions $8^{\prime}$ and $8^{\prime \prime}$ ). The production of the methyl radical and acetylene is believed to proceed through a fourcenter transition state of the allyl radical, although some contribution may be due to dissociation of 1-propenyl radicals.

At $351 \mathrm{~nm}$, the $\tilde{\mathrm{A}}$ state is believed to convert to the groundstate allyl radical through isomerization to the ground-state cyclopropyl radical followed by ring opening. $\mathrm{H}$ atom loss was the only result seen at these energies. RRKM calculations predicted reactions $8^{\prime}$ and $8^{\prime \prime}$ to be the major channels.

Deyerl et al. ${ }^{3,7 a}$ also studied the photodissociation of the allyl radical formed through the pyrolysis of allyl iodide. The allyl radical was excited near $250 \mathrm{~nm}$, and the resulting $\mathrm{H}$ atoms were photoionized and detected using Lyman- $\alpha$ radiation. Several different deuterated allyl iodide compounds were used to determine the site selectivity of $\mathrm{H}$ atom loss from the allyl radical. It was found that there was a preference for the loss of the central hydrogen to form allene (reaction 7). Once again, a second channel was seen, which was believed to be the production of allene or propyne following isomerization to the 2-propenyl radical (reactions $8^{\prime}$ and $8^{\prime \prime}$ ). RRKM calculations predicted the direct formation of allene to be favored by a factor of between 2 and 3 over allene or propyne production via isomerization. It is interesting that there is a discrepancy in the RRKM results between this study and that of Stranges et al.; Stranges et al. used a lower barrier to the isomerization of the allyl radical of $48.6 \mathrm{kcal} / \mathrm{mol}$ whereas Deyerl et al. used their ab initio result of $66 \mathrm{kcal} / \mathrm{mol}$. Deyerl et al.'s RRKM prediction was qualitatively in agreement with their experimental measurement of the relative rates of the direct $\mathrm{H}+$ allene dissociation channel and the product channels from isomerization to 2-propenyl. However, their RRKM prediction ${ }^{7 b}$ for the $\mathrm{H}+$ propyne/H + allene branching ratio from the 2-propenyl radical isomer was $6: 1$, a ratio much higher than that determined from the RRKM parameters in ref 23. 
The study presented here identifies the major reaction pathways of allyl iodide upon excitation at $193 \mathrm{~nm}$ as well as the secondary reaction pathways of the allyl radical product and also validates the higher $(>60 \mathrm{kcal} / \mathrm{mol})$ ab intio predictions for the allyl $\rightarrow$ 2-propenyl isomerization barrier height. The experiments include photofragment translational spectroscopy with two different detection methods and $\mathrm{H}$ atom Rydberg timeof-flight (HRTOF) spectroscopy. As will be described in the Experimental Section, the first photofragment translational spectroscopy apparatus uses the traditional electron impact ionization of the photofragments in its detection scheme. The second uses synchrotron radiation at the Chemical Dynamics Beamline at the Advanced Light Source (ALS), Lawrence Berkeley National Laboratory, as a photoionization source. The latter approach offers several advantages. The existence of secondary dissociation channels can make data analysis difficult. The use of "soft ionization" can minimize photofragment cracking in the ionizer, so, for example, the dominant contribution to the signal at $m / e=40$ is from a neutral mass 40 fragment entering the ionizer rather than from a daughter ion of a higher mass neutral fragment that entered the ionizer. Also, the tunability of the ionization energy helps one distinguish between different isomeric forms of the same mass products. However, the electron impact ionization data were key in detecting the I atom product in either spin-orbit state with equal probability, thus allowing us to predict the velocities of the observed $\mathrm{m} / \mathrm{e}$ $=40$ products resulting from unstable allyl radical $\mathrm{C}-\mathrm{H}$ bond fission.

\section{Experimental Section}

The results presented here were taken on two different rotatable-source, fixed-detector, crossed-laser molecular beam apparatuses and a newly constructed HRTOF apparatus. The first apparatus has been described in detail elsewhere. ${ }^{26}$ Briefly, a beam of allyl iodide $(\sim 10 \%)$ was formed by passing $\mathrm{He}$ through a bubbler containing allyl iodide at room temperature (Aldrich, 98\%) to produce a total backing pressure of 300 Torr and expanding the He through a continuous nozzle of $0.1-\mathrm{mm}$ diameter. The nozzle was heated to $\sim 200{ }^{\circ} \mathrm{C}$ to reduce the formation of clusters. The beam was intersected at right angles by an attenuated 193-nm (ArF transition) Lumonics PM 848 excimer beam focused to a $1.5 \mathrm{~mm} \times 3.0 \mathrm{~mm}$ spot in the interaction region. Typical laser energies were $\sim 4 \mathrm{~mJ} /$ pulse. A small portion of the resulting photofragments scatter within the $1.5^{\circ}$ acceptance angle of the detector and travel $44.4 \mathrm{~cm}$ where they are ionized by $200-\mathrm{eV}$ electron impact ionization, selected according to their mass/charge ratio by a quadrupole mass filter, and detected by a Daly detector. ${ }^{27}$ Signal collection is triggered by the laser pulse and recorded as a function of time. The times of flight in the spectra shown are the sums of neutral and ion flight times, but the fits account for the ion flight times by using a previously determined ion flight constant of $4.5 \mu \mathrm{s}(\mathrm{amu})^{-1 / 2}$. The parent beam velocity was characterized by directing the molecular beam on axis and raising a chopper wheel in the beam path. A typical peak molecular beam velocity in the number density distribution, $N(v)$, was $8.0 \times 10^{4} \mathrm{~cm} / \mathrm{s}$ with a velocity spread $\left(\Delta v / v_{\text {peak }}\right)$ of $22 \%$ in which $\Delta v$ was calculated from the full width at half-maximum, fwhm, of $N(v)$.

The second apparatus is located at the Advanced Light Source at Lawrence Berkeley National Laboratory and has also been previously described. ${ }^{28} \mathrm{He}$ was bubbled through room-temperature allyl iodide to produce a total backing pressure of $~ 500$ Torr. The sample was then expanded through a piezo ProchTrick $^{29}$ pulsed nozzle with a $1-\mathrm{mm}$ diameter orifice heated to a temperature of $\sim 80{ }^{\circ} \mathrm{C}$. The beam was crossed with an attenuated beam from a Lambda Physik LPX-200 excimer operating at $193 \mathrm{~nm}$ and an energy of $\sim 4 \mathrm{~mJ} /$ pulse and focused to $2 \mathrm{~mm} \times 4 \mathrm{~mm}$ spot. The resulting neutral photofragments travel $15.2 \mathrm{~cm}$ and are ionized using tunable synchrotron radiation and pass through a quadrupole mass filter to a Daly detector. The synchrotron radiation passes through an Ar filter for all data as well as a $\mathrm{MgF}_{2}$ filter for data taken at an ionization energy of less than $10.8 \mathrm{eV}$ to reduce the transmission of highenergy harmonics. For most of the spectra presented, the photoionization source had a bandwidth near 5\%, and the photoionization efficiency data were collected at a bandwidth of $\sim 3 \%$. The parent molecular velocity was characterized by directing the molecular beam on axis and analyzing the "hole" formed in the signal at $m / e=41$ (allyl radical). A typical peak molecular beam velocity in the distribution $N(v)$ was $12.6 \times$ $10^{4} \mathrm{~cm} / \mathrm{s}$ with a fwhm of $22 \%$. An ion flight constant of 10.8 $\pm 0.1 \mu \mathrm{s}(\mathrm{amu})^{-1 / 2}$ was used to correct for ion flight time. This value was determined by varying the constant for both $\mathrm{m} / \mathrm{e}=$ $127\left(\mathrm{I}^{+}\right)$photodissociation data taken at $10.8 \mathrm{eV}$ and parent beam time-of-flight measurements until a good fit to the leading edge was obtained using a translational energy distribution $\left(P\left(E_{\mathrm{T}}\right)\right)$ that was known to fit prior $m / e=127$ measurements that were obtained using the electron impact ionization machine.

Finally, HRTOF ${ }^{30,31}$ was used to characterize the $\mathrm{H}$ loss channels. Briefly, He was bubbled through room-temperature allyl iodide to produce a total backing pressure of 300 Torr and expanded through a 500- $\mu \mathrm{m}$ diameter orifice pulsed valve (General Valve Iota One) that had been heated to $80{ }^{\circ} \mathrm{C}$. The sample passed through a $600-\mu \mathrm{m}$ diameter skimmer and traveled roughly $5 \mathrm{~cm}$ to the interaction region, where it was intersected with a $1-\mathrm{mJ} /$ pulse attenuated beam from a Lambda Physik EMG-103-MSC excimer operating at $193 \mathrm{~nm}$. The resulting $\mathrm{H}$ atoms were crossed with 121.6-nm light to promote a $1 \mathrm{~s} \rightarrow 2 \mathrm{p}$ (Lyman- $\alpha$ ) transition. This light was obtained by doubling the 729.8-nm output of a Nd:YAG (Continuum PowerLite 9020) pumped dye laser (Lambda Physik FL3002) in a KDP crystal and tripling the resulting 364.9-nm light in 42 mbar of $\mathrm{Kr}$. The $2 \mathrm{p} \mathrm{H}$ atom was then excited to a Rydberg state via 366-nm light formed by doubling the output of a Lambda Physik FL3002E dye laser (also pumped by the Continuum PowerLite 9020). A field was maintained in the interaction region by applying a $-20.0 \mathrm{~V}$ potential to a disk $0.5 \mathrm{~cm}$ below the interaction region. The Rydberg $\mathrm{H}$ atoms that scattered $90^{\circ}$ with respect to the molecular beam axis traveled $42.35 \mathrm{~cm}$ and were ionized in the field between a wire mesh held at $+20.0 \mathrm{~V}$ and a Burle TOF-4000 detector at a potential of $-2.0 \mathrm{kV}$ that were separated by $\sim 1 \mathrm{~mm}$. Signal was recorded with respect to the Nd:YAG pump pulse by a Stanford SR430 MCS. It was not necessary to characterize the parent beam velocity because it is orders of magnitude smaller than the velocity of the $\mathrm{H}$ atoms, yielding no apparent difference in the fit obtained from the center of mass to lab frame conversion when changing the initial velocity of the parent beam from $3 \times 10^{4}$ to $13 \times 10^{4}$ $\mathrm{cm} / \mathrm{s}$.

We found no evidence of clusters when looking for signal at $m / e=168$ (parent mass) at a $10^{\circ}$ source angle; data taken at smaller source angles had a cluster contribution that was detected at the parent mass. Data previously taken at a nozzle temperature of $50{ }^{\circ} \mathrm{C}$ showed evidence of a cluster contribution at $\mathrm{m} / \mathrm{e}=$ 40 .

\section{Results and Analysis}

Figure 1 shows the time-of-flight spectrum (TOF) of I atoms $(m / e=127)$ taken at a source angle of $15^{\circ}$ with respect to the 


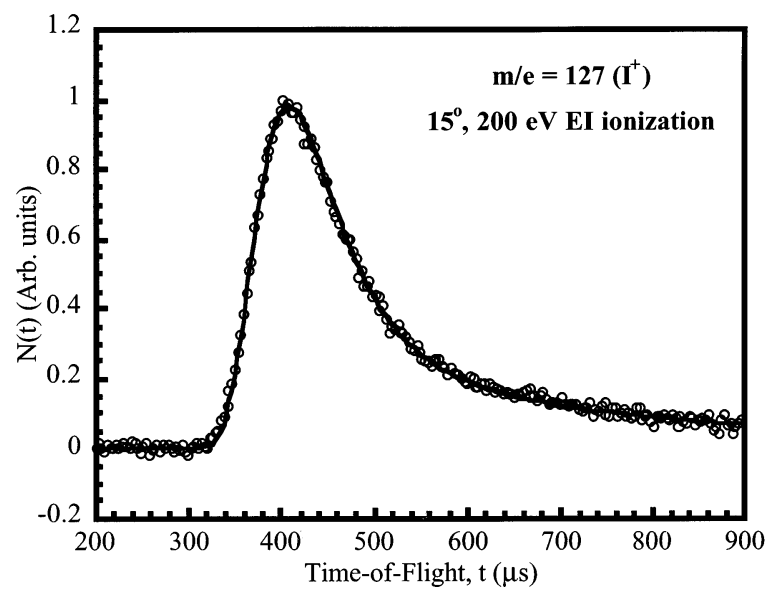

Figure 1. TOF spectrum of $m / e=127\left(\mathrm{I}^{+}\right)$resulting from the photodissociation of allyl iodide using $200-\mathrm{eV}$ electron impact ionization at a source angle of $15^{\circ}$. The open circles are experimental data, and the solid line is the forward convolution fit using the $P\left(E_{\mathrm{T}}\right)$ shown in Figure 2.

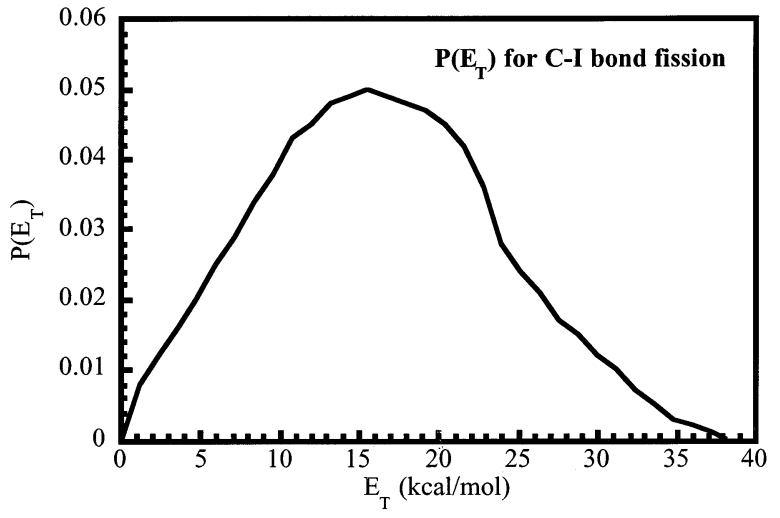

Figure 2. $P\left(E_{\mathrm{T}}\right)$ for $\mathrm{C}-\mathrm{I}$ bond fission, forming both $\mathrm{I}\left({ }^{2} \mathrm{P}_{1 / 2}\right)$ and $\mathrm{I}\left({ }^{2} \mathrm{P}_{3 / 2}\right)$, in allyl iodide derived from the forward convolution fitting of the $m / e$ $=127\left(\mathrm{I}^{+}\right)$spectrum shown in Figure 1.

detector axis. The I atoms in this spectrum were ionized using 200-eV electron impact ionization. As shall be explained later, the signal, shown in open circles, is composed of two overlapping contributions because the electron impact ionization detects both the $\mathrm{I}\left({ }^{2} \mathrm{P}_{3 / 2}\right)$ and $\mathrm{I}\left({ }^{2} \mathrm{P}_{1 / 2}\right)$ formation channels (reactions 1 and 2 ). Figure 2 shows the $\mathrm{C}-\mathrm{I}$ bond fission recoil translationalenergy distribution, $P\left(E_{\mathrm{T}}\right)$, derived from the forward convolution fit to the $m / e=127$ data shown as a solid line in Figure 1. The $P\left(E_{\mathrm{T}}\right)$ peaks near $16 \mathrm{kcal} / \mathrm{mol}$ and extends to $39 \mathrm{kcal} / \mathrm{mol}$.

To look for evidence of the $\mathrm{I}\left({ }^{2} \mathrm{P}_{1 / 2}\right)$ channel, we took advantage of the tunability of the synchrotron radiation used for the photoionization of products at the ALS and re-collected the $m / e=127$ data using a photoionization energy of $9.5 \mathrm{eV}$, which is much less than the $\mathrm{I}\left({ }^{2} \mathrm{P}_{3 / 2}\right)$ ionization energy of 10.45 $\mathrm{eV} .{ }^{32}$ This TOF signal, shown in Figure 3, is thus only from the $\mathrm{I}\left({ }^{2} \mathrm{P}_{1 / 2}\right)$ channel, and the observed recoil velocities of the $\mathrm{I}\left({ }^{2} \mathrm{P}_{1 / 2}\right)$ atoms in this spectrum are different than the distribution of recoil velocities for all I atom products that fit the I atom spectrum when electron impact was used. This shows that the photodissociation of allyl iodide at $193 \mathrm{~nm}$ has two allyl + I primary product channels, one producing $\mathrm{I}\left({ }^{2} \mathrm{P}_{3 / 2}\right)$ and the other producing $\mathrm{I}\left({ }^{2} \mathrm{P}_{1 / 2}\right)$. (We do not fit the slow tail visible from $300-$ $400 \mu$ s because it is likely due to the dissociation of clusters.) The $P\left(E_{\mathrm{T}}\right)$ derived from the forward convolution fit to the $\mathrm{I}\left({ }^{2} \mathrm{P}_{1 / 2}\right)$ data in Figure 3 is the solid line shown in Figure 4 and is noticeably different from the $P\left(E_{\mathrm{T}}\right)$ used to fit $m / e=127$ at an ionization energy of $200 \mathrm{eV}$, as shown in Figure 2. It has a

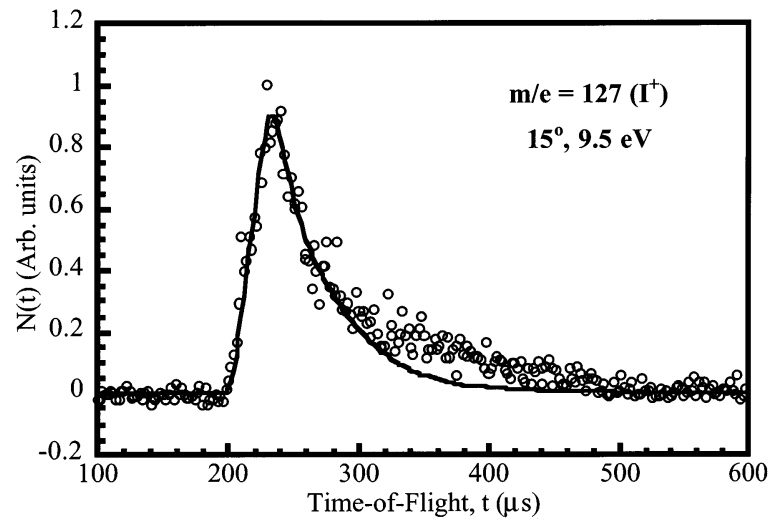

Figure 3. TOF spectrum of $m / e=127\left(\mathrm{I}^{+}\right)$due to the $\mathrm{I}\left({ }^{2} \mathrm{P}_{1 / 2}\right)$ formation channel only, taken at a photoionization energy of $9.5 \mathrm{eV}$ and a source angle of $15^{\circ}$. The open circles are experimental data, and the solid line is the forward convolution fit using the solid $P\left(E_{\mathrm{T}}\right)$ shown in Figure 4.

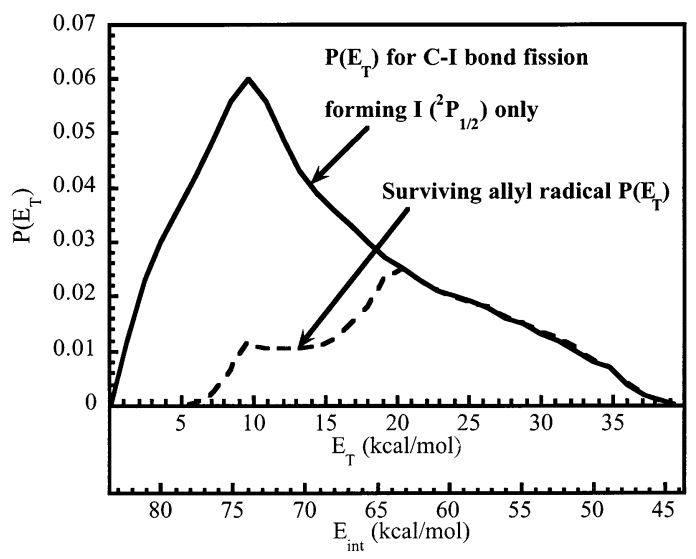

Figure 4. $P\left(E_{\mathrm{T}}\right)$ for all primary $\mathrm{C}-\mathrm{I}$ bond fission events forming $\mathrm{I}$ atoms in the spin-orbit excited ${ }^{2} \mathrm{P}_{1 / 2}$ state $(-)$ and $P\left(E_{\mathrm{T}}\right)$ for primary $\mathrm{C}-\mathrm{I}$ bond fission forming $\mathrm{I}\left({ }^{2} \mathrm{P}_{1 / 2}\right)+$ allyl radicals that are stable to secondary dissociation $(---)$. The solid line $P\left(E_{\mathrm{T}}\right)$ is derived from the forward convolution fitting of $m / e=127$ data taken at an ionization energy of $9.5 \mathrm{eV}$ (Figure 3). The top $x$ axis is the translational energy of the recoiling fragments, and the bottom $x$ axis is the corresponding internal energy of the nascent allyl radical. The dashed-line $P\left(E_{\mathrm{T}}\right)$ is derived from the forward convolution fit to the $m / e=41$ data taken at a source angle of $30^{\circ}$ and an ionization energy of $9.5 \mathrm{eV}$ (Figure 5).

faster rising edge peaking near $10 \mathrm{kcal} / \mathrm{mol}$ and extends to 39 $\mathrm{kcal} / \mathrm{mol}$. (Note that at a photoionization energy of $10.8 \mathrm{eV}$, which is above the ionization limit of both $\mathrm{I}\left({ }^{2} \mathrm{P}_{3 / 2}\right)$ and $\mathrm{I}\left({ }^{2} \mathrm{P}_{1 / 2}\right)$, one does not detect the atoms with a probability that is independent of the state; we return to this point in the Discussion.)

The $P\left(E_{\mathrm{T}}\right)$ 's in Figures 2 and 4 along with eq 6 were used to calculate the range of internal energies present in the photofragments. From eq 6, the energy available for the translational and internal energy of the radical formed in the $\mathrm{I}\left({ }^{2} \mathrm{P}_{3 / 2}\right)$ channel is $105.3 \mathrm{kcal} / \mathrm{mol}$. The translational energy ranges from $0-39$ $\mathrm{kcal} / \mathrm{mol}$, and the internal energy of the allyl radical ranges from $105.3-66.3 \mathrm{kcal} / \mathrm{mol}$. Likewise, the allyl radicals from the $\mathrm{I}\left({ }^{2} \mathrm{P}_{1 / 2}\right)$ channel have an internal energy range of $83.6-44.6 \mathrm{kcal} /$ mol. Thus, if one assumes a barrier height of $60 \mathrm{kcal} / \mathrm{mol}$ for the dissociation of the allyl radical to produce allene $+\mathrm{H}$ (reaction 7), any stable allyl radical detected at $m / e=41$ is likely to be produced from the $\mathrm{I}\left({ }^{2} \mathrm{P}_{1 / 2}\right)+\mathrm{C}_{3} \mathrm{H}_{5}$ product channel because all the allyl radicals from the $\mathrm{I}\left({ }^{2} \mathrm{P}_{3 / 2}\right)+\mathrm{C}_{3} \mathrm{H}_{5}$ product channel have internal energy that is higher than $60 \mathrm{kcal} / \mathrm{mol}$.

The TOF spectrum of $m / e=41$ at $30^{\circ}$ taken at an ionization energy of $9.5 \mathrm{eV}$ is shown in Figure 5. As expected, the peak 


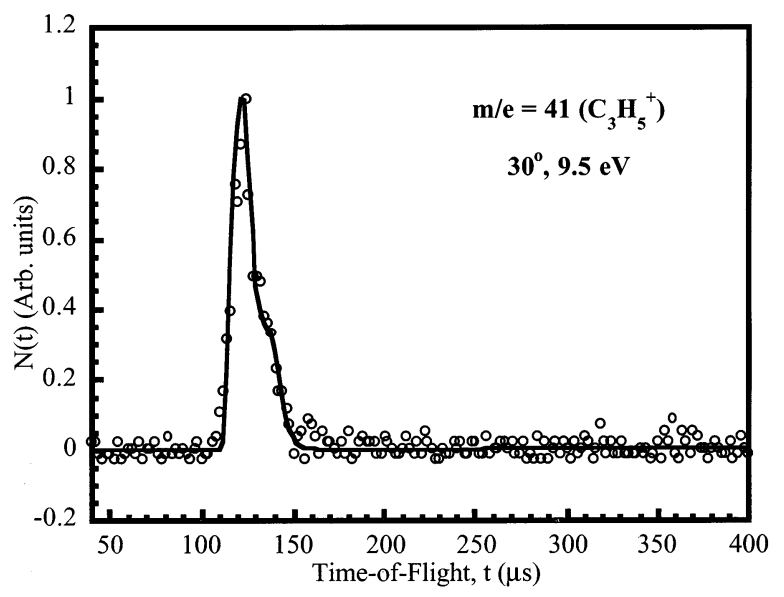

Figure 5. TOF spectrum of $m / e=41\left(\mathrm{C}_{3} \mathrm{H}_{5}{ }^{+}\right)$taken at a photoionization energy of $9.5 \mathrm{eV}$ and a source angle of $30^{\circ}$. The $P\left(E_{\mathrm{T}}\right)$ derived from the forward convolution fit to these data is the dashed line in Figure 4.

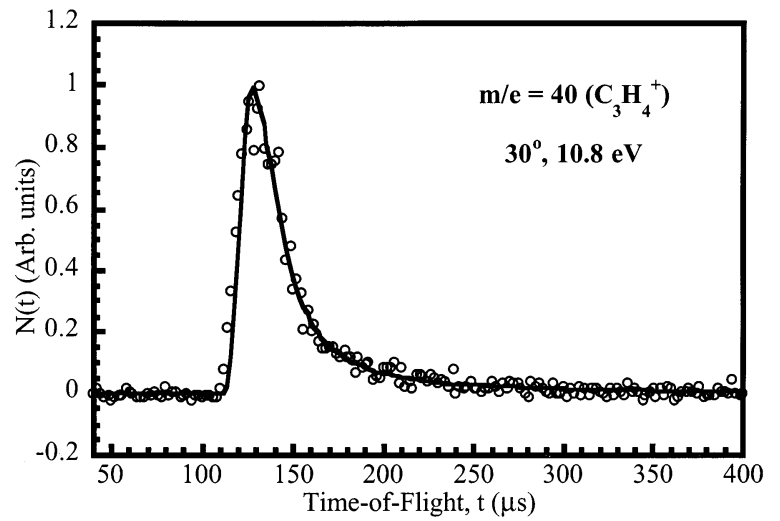

Figure 6. TOF spectrum of $m / e=40\left(\mathrm{C}_{3} \mathrm{H}_{4}{ }^{+}\right)$taken at a photoionization energy of $10.8 \mathrm{eV}$ and a source angle of $30^{\circ}$. The $\mathrm{m} / \mathrm{e}=40$ peak arises from secondary dissociation of the allyl radical and is fit with the $\mathrm{C}-\mathrm{I}$ bond fission $P\left(E_{\mathrm{T}}\right)$ (Figure 2) because the $\mathrm{C}-\mathrm{H}$ bond fission in the radicals imparts little additional translational energy to the heavy mass 40 product.

is visibly narrower than that predicted from the $\mathrm{I}\left({ }^{2} \mathrm{P}_{1 / 2}\right)$ channel $P\left(E_{\mathrm{T}}\right)$ (Figure 4, solid line) because many of the allyl radicals produced in the $\mathrm{I}\left({ }^{2} \mathrm{P}_{1 / 2}\right)$ channel have internal energy high enough to undergo secondary dissociation. What was found, however, is that the $m / e=41$ signal extends to much lower translational energies than would be predicted if all of the allyl radicals with internal energy values higher than the $60 \mathrm{kcal} /$ mol barrier dissociated. The spectrum in Figure 5 was fit using the $P\left(E_{\mathrm{T}}\right)$ shown in Figure 4 (dashed line); using a $P\left(E_{\mathrm{T}}\right)$ that drops to 0 at $E_{\mathrm{T}}=23.6 \mathrm{kcal} / \mathrm{mol}$ did not give a suitable fit to the data (i.e., assuming that all allyl radicals with internal energy values greater than $60 \mathrm{kcal} / \mathrm{mol}$ would dissociate and not be detected at $m / e=41$ ). As will be seen in the Discussion, the marked stability of the allyl radical with greater than $60 \mathrm{kcal} /$ mol internal energy is due to a centrifugal barrier ${ }^{33,34}$ to dissociation of the highly rotationally excited allyl radical.

Data collected at $m / e=40\left(\mathrm{C}_{3} \mathrm{H}_{4}{ }^{+}\right)$at an angle of $30^{\circ}$ with an ionization energy of $10.8 \mathrm{eV}$ are shown in Figure 6. Because the loss of an $\mathrm{H}$ atom will impart negligible translational energy to its sibling fragment, the spectrum shown in Figure 6 was fit using the same $P\left(E_{\mathrm{T}}\right)$ derived from the forward convolution fit to the $m / e=127$ electron impact ionization spectra (Figure 2). The fact that the signal and fit agree on the fast edge reinforces the belief that there must be an $\mathrm{I}\left({ }^{2} \mathrm{P}_{3 / 2}\right)$ contribution because any $m / e=41$ from an $\left.\mathrm{I}^{2} \mathrm{P}_{1 / 2}\right)$ channel at such high translational

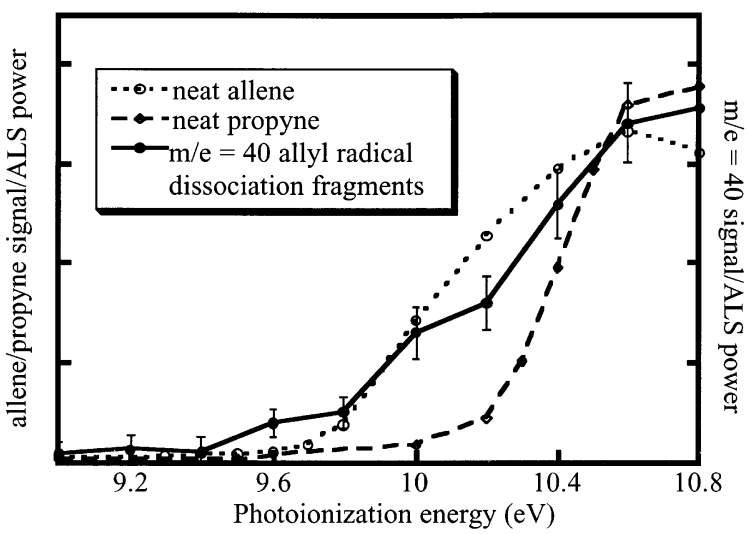

Figure 7. Photoionization efficiency (PIE) curve for the products from allyl iodide giving a signal at $m / e=40\left(\mathrm{C}_{3} \mathrm{H}_{4}{ }^{+}\right)$. The signal accumulated in 60000 laser shots in the TOF spectrum at a source angle of $15^{\circ}$ at each photoionization energy is integrated from $100-140-\mu$ s flight times, normalized with the measured ALS power, and shown with solid circles. (Although these data were taken at a nozzle temperature of $50{ }^{\circ} \mathrm{C}$, integrating to $200 \mu$ s gave the same PIE curve.) The data points are shown with error bars and with straight-line extrapolations to guide the eye. PIE curves of neat allene (-- $\bigcirc--)$ and neat propyne $(--\diamond--)$ taken under similar conditions are provided for comparison and are reproduced with permission from the work of Mueller et al. ${ }^{41}$ All data shown were taken with the $\mathrm{MgF}_{2}$ window. The allene and propyne curves are plotted with actual integrated signals (normalized with ALS power), and the $m / e=40$ product signal is scaled arbitrarily to the allene and propyne curves.

energies would not have enough internal energy to undergo secondary dissociation to give $m / e=40$ products.

A photoionization efficiency (PIE) curve of the $m / e=40$ signal (solid line) is shown in Figure 7 along with PIE curves of both allene (dotted line) and propyne (dashed line) (allene and propyne curves reproduced with permission from ref 41). The appearance of signal near $9.6 \mathrm{eV}$ and the slow rise of the signal with ionization energy show that the dominant path of dissociation of the allyl radical results in the formation of allene $(\mathrm{IE}=9.7 \mathrm{eV}),{ }^{32}$ although the formation of a small amount of propyne $(\mathrm{IE}=10.36 \mathrm{eV})^{32}$ cannot be ruled out entirely. The fact that there is not much of a red shift in the ionization energy of allene indicates that the allene product is not highly vibrationally excited. Previous PIE curves of $m / e=40$ taken in the photodissociation of allyl chloride at $193 \mathrm{~nm}$ were redshifted by as much as $1 \mathrm{eV}$ or more ${ }^{35}$ This is not surprising because allyl chloride photodissociation includes a significant $\mathrm{HCl}$ elimination channel, producing $m / e=40$ fragments with up to $124 \mathrm{kcal} / \mathrm{mol}$ of available energy. ${ }^{35}$ As will be discussed, allyl iodide does not undergo significant $\mathrm{HI}$ elimination, so the $m / e=40$ products are the result of secondary dissociation of nascent allyl radicals and have an available energy near $50 \mathrm{kcal} /$ mol, much of which is partitioned to $\mathrm{H}$ atom translational energy. Thus, one expects the $m / e=40$ products to have modest internal energy, and the $m / e=40 \mathrm{PIE}$ curve should then not be heavily red-shifted from that of cold $\mathrm{C}_{3} \mathrm{H}_{4}$ radicals. The extent of allene and propyne contributions to the $m / e=40$ channel can also be examined by taking note of the difference in ionization energy of allene $(9.7 \mathrm{eV})^{32}$ and propyne $(10.36 \mathrm{eV})^{32}$ and comparing $\mathrm{m} / \mathrm{e}=40$ spectra taken at ionization energies of 10.0 and $10.8 \mathrm{eV}$. Figure 8 shows overlaid spectra taken at ionization energies of 10.0 (triangles) and $10.8 \mathrm{eV}$ (circles) with a nozzle temperature of $50{ }^{\circ} \mathrm{C}$. Although at this temperature the slow signal has some cluster contribution, the fact that both peaks agree on the fast edge shows that any propyne contribution to the $m / e=40$ signal must be minimal at internal energies close to the dissociation threshold. This is the critical internal- 


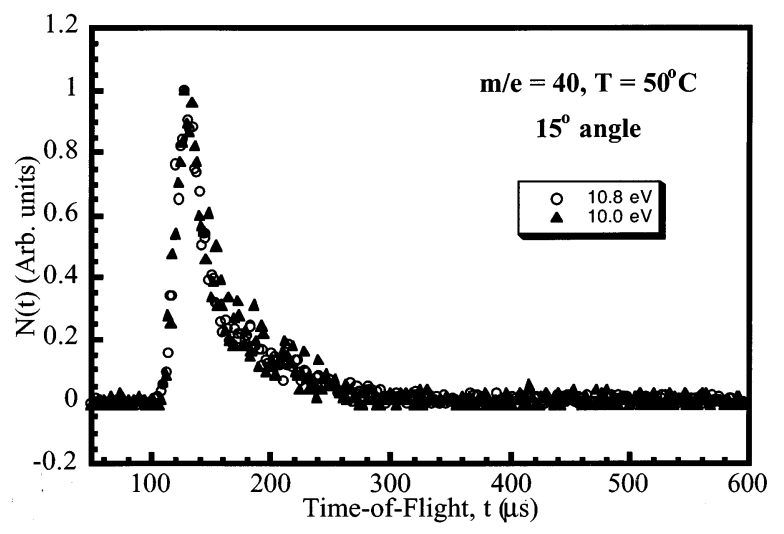

Figure 8. TOF spectra at $m / e=40$ taken at $10.0 \mathrm{eV}(\mathbf{\Delta})$ and $10.8 \mathrm{eV}$ (O) at a source angle of $15^{\circ}$ and a nozzle temperature of $50{ }^{\circ} \mathrm{C}$. Because the ionization energies of allene and propyne are 9.7 and $10.36 \mathrm{eV}$, respectively, comparing the two spectra should help detect the appearance of any propyne contribution in the $m / e=40$ spectra.

energy region for distinguishing between the experimentally determined allyl $\rightarrow$ 2-propenyl isomerization barrier of 48.6 $\mathrm{kcal} / \mathrm{mol}$ and the higher theoretical prediction of the barrier, $>60 \mathrm{kcal} / \mathrm{mol}$. We return to this point in the Discussion.

Signal was also found at $m / e=39\left(\mathrm{C}_{3} \mathrm{H}_{3}{ }^{+}\right)$. Although the allyl radical does have an $\mathrm{H}_{2}$ elimination channel that produces the propargyl radical $(m / e=39)$, it should proceed via too high a barrier to be possible in these experiments, so some of this signal could be from dissociative ionization. The appearance of signal started near $9 \mathrm{eV}$, which is a much lower energy than the $11.48-\mathrm{eV}$ appearance energy for $\mathrm{C}_{3} \mathrm{H}_{3}$ formation from allene. ${ }^{32}$ Thus, whereas the slow contribution of the $m / e=39$ signal could be due to dissociative ionization of allene, it is not energetically possible for the fast products. The fast signal might be due to a minor $\mathrm{HI}$ or $\mathrm{H}_{2}$ elimination channel followed by secondary dissociation that was not discernible in the $m / e=$ 127 spectrum. The possibility of $\mathrm{HI}$ elimination (reaction 3) was investigated by looking for signal at $m / e=128$, taking 75000 and 150000 shots at ionization energies of 10.2 and $12.0 \mathrm{eV}$, respectively, at an angle of $15^{\circ}$ and $1 \times 10^{6}$ shots at a $10^{\circ}$ angle using $200-\mathrm{eV}$ electron impact ionization. There was no signal found.

Following the initial dissociation, the allyl radical product has enough internal energy potentially to isomerize to the 1-propenyl radical and cleave a $\mathrm{C}-\mathrm{C}$ bond, forming mass 26 $(\mathrm{H}-\mathrm{C} \equiv \mathrm{C}-\mathrm{H})$ and mass $15\left(\mathrm{CH}_{3}\right)$ fragments (reaction 9'). Signal was found at $m / e=26$, but unlike the $\mathrm{C}-\mathrm{C}$ fission signal we have detected using the same technique from the dissociation of the 1-propenyl radical, ${ }^{36}$ the signal here is too fast to be energetically allowed from secondary dissociation of the allyl radical that is formed in a one-photon dissociation of the precursor. At the translational energies observed for $m / e=26$, it is energetically possible to form $\mathrm{C}_{2} \mathrm{H}_{2}$ from $\mathrm{CH}_{3} \mathrm{I}$ elimination of allyl iodide (reaction 4). This explanation is doubtful, however, because $\mathrm{CH}_{3} \mathrm{I}$ is known to crack during electron impact ionization to produce I atoms. Indeed, the data in Figure 1 could be fit without involving a $\mathrm{CH}_{3} \mathrm{I}$ elimination channel, and the $m / e=127$ fit is momentum-matched with $m / e=40$ from the unimolecular dissociation of the $\mathrm{C}_{3} \mathrm{H}_{5}$ cofragment from $\mathrm{C}-\mathrm{I}$ bond fission only. Thus, production of $m / e=26$ is most likely due to a multiphoton process. Signal at $m / e=15$ was found as well, but it was easily fit with the $m / e=40 P\left(E_{\mathrm{T}}\right)$ (Figure 2), suggesting dissociative ionization of the $m / e=40$ product. Thus, the isomerization of allyl to the 1-propenyl radical at the internal energies present in this study does not compete effectively with the direct $\mathrm{H}+$ allene bond-fission channel.

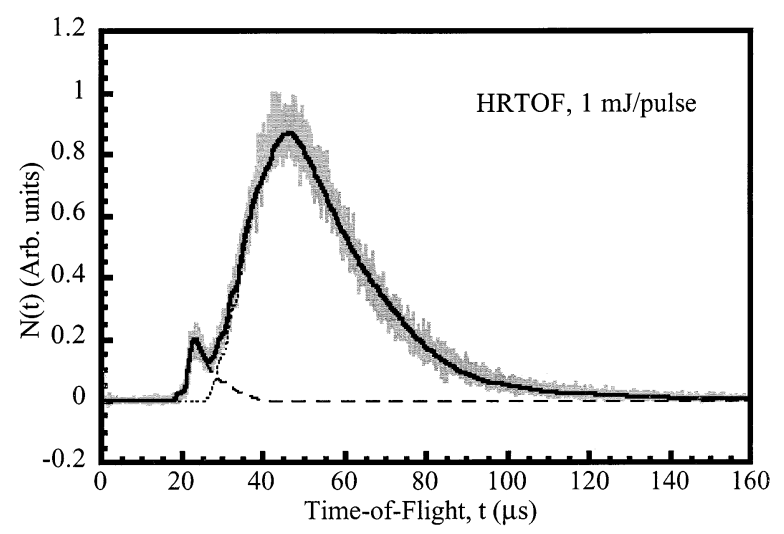

Figure 9. HRTOF spectrum of allyl iodide taken at a photolysis energy of $1 \mathrm{~mJ} /$ pulse. The first peak $(---)$ is attributed to primary $\mathrm{C}-\mathrm{H}$ bond fission, whereas the second peak $(\cdots)$, which is slower than the first, is attributed to $\mathrm{H}$ atoms from the dissociation of the allyl radical formed in the primary photolysis of allyl iodide. The $P\left(E_{\mathrm{T}}\right)$ 's derived from the forward convolution fitting of this spectrum are shown in Figures 10 and 11.

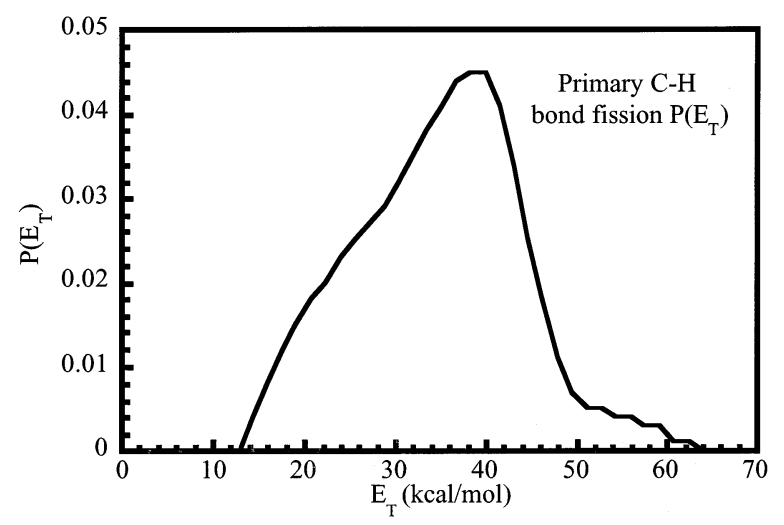

Figure 10. $P\left(E_{\mathrm{T}}\right)$ derived from the forward convolution fitting of the fast $\mathrm{H}$ atom signal in Figure 9 attributed to primary $\mathrm{C}-\mathrm{H}$ bond fission in allyl iodide $(---)$. It gives the dashed contribution to the signal in the $m / e=167\left(\mathrm{C}_{3} \mathrm{H}_{4} \mathrm{I}^{+}\right)$spectrum shown in Figure 13 that is attributed to the momentum-matched partner fragment.

The work here also characterizes the velocity distribution of the $\mathrm{H}$ atoms from the primary and secondary $\mathrm{H}$ loss channels. Figure 9 shows the HRTOF spectrum of $\mathrm{H}$ atoms from allyl iodide. There are two obvious peaks present, a small fast peak and a large slower peak. A study was undertaken to determine the power dependence of the signal. Data were collected at 0.25-, $0.5-$, and $1.0-\mathrm{mJ} /$ pulse photolysis energies, taken in random 5000 shot intervals, and then summed. It was found that peak area scaled roughly linearly with photolysis power and that the ratio of the small fast peak to the large slow peak stayed constant as the laser power was quadrupled, indicating that both channels occur via a one-photon process. Figures 10 and 11 display the $P\left(E_{\mathrm{T}}\right)$ 's used to fit the data shown in Figure 9. The fast $\mathrm{H}$ atom signal $P\left(E_{\mathrm{T}}\right)$ (Figure 10 ) peaks near $40 \mathrm{kcal} / \mathrm{mol}$ and extends to $\sim 62 \mathrm{kcal} / \mathrm{mol}$ and is attributed to a primary $\mathrm{H}$ atom loss channel of allyl iodide. The small, high-energy shoulder may be due to a multiphoton process because it is hard to discern the peak for all photolysis energies given the signal-to-noise ratio at lower energies. As shown in Figure 11, the slow $\mathrm{H}$ atom signal attributed to secondary $\mathrm{H}$ atom loss still imparts significant energy into product translation, peaking near $8 \mathrm{kcal} / \mathrm{mol}$ and extending to $\sim 28 \mathrm{kcal} / \mathrm{mol}$.

Signal was also found at the parent mass formed when allyl iodide loses an $\mathrm{H}$ atom $\left(m / e=167\left(\mathrm{C}_{3} \mathrm{H}_{4} \mathrm{I}^{+}\right)\right)$using $200-\mathrm{eV}$ electron impact ionization corresponding to the primary $\mathrm{H}$ loss 


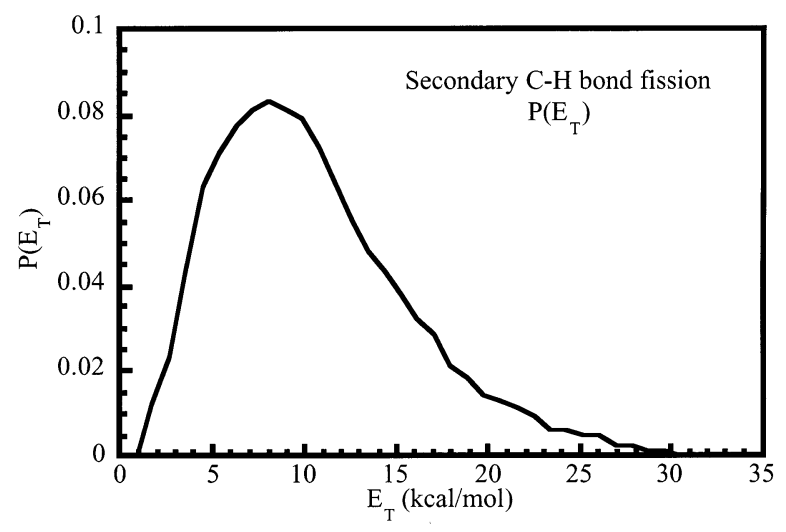

Figure 11. $P\left(E_{\mathrm{T}}\right)$ derived from forward convolution fitting of the slower $\mathrm{H}$ atom signal in Figure $9(\cdots)$ attributed to $\mathrm{H}$ atoms from the secondary dissociation of allyl iodide that produces allene $+\mathrm{H}$.

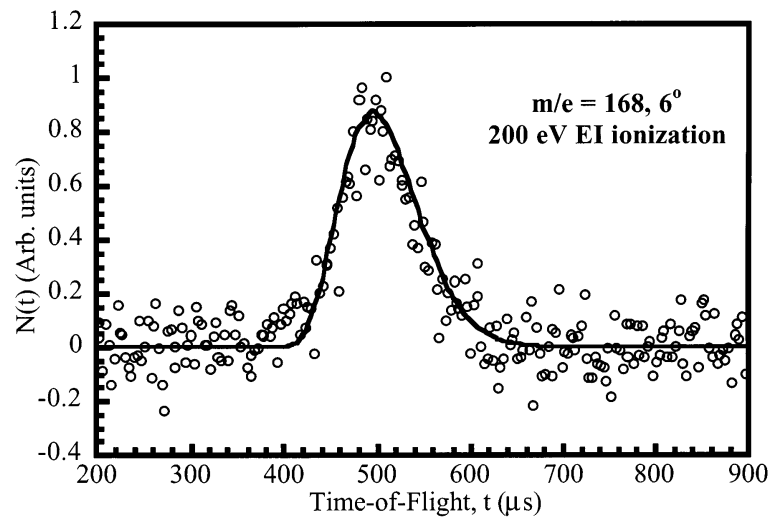

Figure 12. TOF spectrum of $m / e=168$ (allyl iodide) taken at a $6^{\circ}$ source angle and 200-eV EI ionization because of the photodissociation of clusters. The mass 168 cluster fragments can crack to produce $m / e$ $=167$ and 127, which therefore complicates the identification of the heavy cofragment signal from the primary $\mathrm{H}$ loss channel spectrum (Figure 13).

channel. This signal was complicated because of clusters at the low angles necessary to detect the slow-moving $\mathrm{C}_{3} \mathrm{H}_{4} \mathrm{I}$ fragment. Data were taken at the parent mass $(m / e=168)$ and a source angle of $6^{\circ}$ to characterize any cluster contribution to the $m / e$ $=167$ signal. Figure 12 shows $m / e=168$ data at a source angle of $6^{\circ}$. Data taken at $m / e=167\left(\mathrm{C}_{3} \mathrm{H}_{4} \mathrm{I}^{+}\right)$and an angle of $6^{\circ}$ are shown in Figure 13. This signal may be due to both the primary $\mathrm{H}$ loss channel and parent $(m / e=168)$ fragments from cluster dissociation cracking in the ionizer. Therefore, the data in Figure 13 were fit using the $P(v)$ because of clusters (dotted line) and the momentum-matched $P\left(E_{\mathrm{T}}\right)$ (dashed line) from the HRTOF primary $\mathrm{H}$ atom loss channel (Figure 10). Both allyl iodide from cluster dissociation and $\mathrm{C}_{3} \mathrm{H}_{4} \mathrm{I}$ products from primary $\mathrm{C}-\mathrm{H}$ fission can crack in the ionizer to produce $\mathrm{I}^{+}(\mathrm{m} / \mathrm{e}=127)$. Therefore, data were collected at $m / e=127$ and source angles of $6^{\circ}$ and $7^{\circ}$ with $200-\mathrm{eV}$ electron impact ionization. These data were fit with the $\mathrm{C}-\mathrm{I}$ bond fission $P\left(E_{\mathrm{T}}\right)$ (Figure 2) along with the cluster $P(v)$ and primary $\mathrm{H}$ loss $P\left(E_{\mathrm{T}}\right)$. The results are shown in Figure 14.

\section{Discussion}

The data here show that $\mathrm{C}-\mathrm{I}$ bond fission of allyl iodide at $193 \mathrm{~nm}$ produces both $\mathrm{I}\left({ }^{2} \mathrm{P}_{3 / 2}\right)$ and $\mathrm{I}\left({ }^{2} \mathrm{P}_{1 / 2}\right)$. Because $\mathrm{I}\left({ }^{2} \mathrm{P}_{3 / 2}\right)$ has an ionization energy of $10.45 \mathrm{eV},{ }^{32}$ the spectrum collected at $9.5 \mathrm{eV}$ corresponds to the $\mathrm{I}\left({ }^{2} \mathrm{P}_{1 / 2}\right)$ signal only. Data taken using $200-\mathrm{eV}$ electron impact ionization were then collected at $\mathrm{m} / \mathrm{e}$ $=127$ to detect an I atom in either spin-orbit state with equal

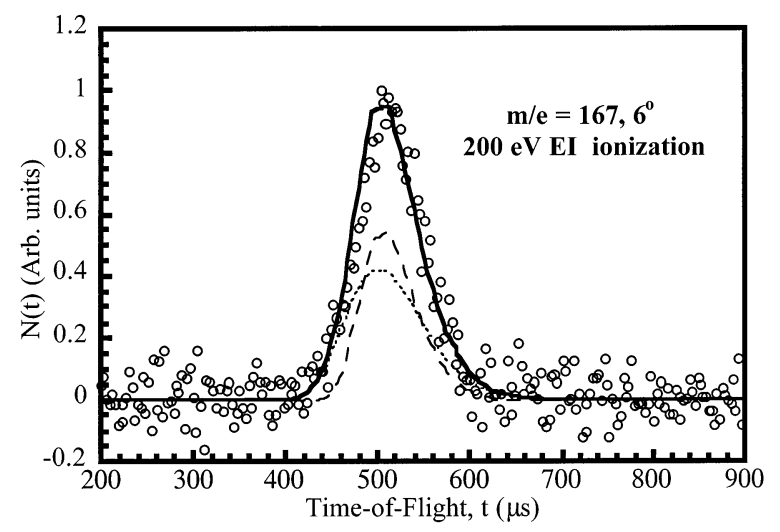

Figure 13. TOF spectrum of $m / e=167\left(\mathrm{C}_{3} \mathrm{H}_{4} \mathrm{I}^{+}\right)$. The signal includes contributions from the primary $\mathrm{C}-\mathrm{H}$ bond fission channel in allyl iodide (dashed line) and $m / e=167$ produced from cracking of the parent allyl iodide from the photodissociation of clusters $(\cdots)$. The dashedline contribution is calculated from the $P\left(E_{\mathrm{T}}\right)$ in Figure 10 because that signal must be momentum-matched with the faster $\mathrm{H}$ atom signal in Figure 9. The cluster contribution to the fit $(\cdots)$ is calculated from the $P(v)$ derived from the forward convolution fitting of the $m / e=$ 168 spectrum shown in Figure 12.

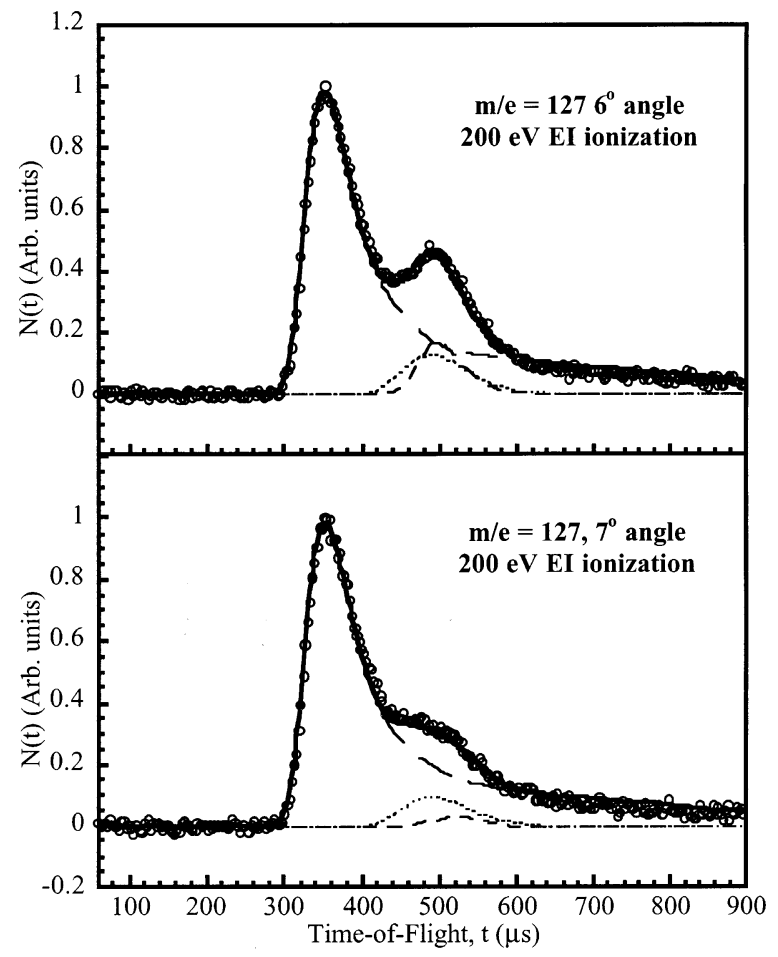

Figure 14. TOF spectra of the signal at $m / e=127$ taken at source angles of $6^{\circ}$ (top panel) and $7^{\circ}$ (bottom panel) using 200-eV EI ionization. The dashed line contribution is calculated using the $P\left(E_{\mathrm{T}}\right)$ shown in Figure 10 that was derived from the forward convolution fitting of the faster peak in the HRTOF $\mathrm{H}$ atom spectrum (Figure 9). The dotted line was calculated using the $P(v)$ derived from forward convolution fitting of the $m / e=168$ spectrum shown in Figure 12 . The fit shown by the long dashed line that fits the main peak in the spectrum is calculated from $P\left(E_{\mathrm{T}}\right)$ for $\mathrm{C}-\mathrm{I}$ bond fission (Figure 2).

probability. It is interesting to compare that spectrum with the data collected at an ionization energy of $10.8 \mathrm{eV}$. Although 10.8 $\mathrm{eV}$ is above the $\mathrm{I}\left({ }^{2} \mathrm{P}_{1 / 2}\right)$ ionization energy and just above the $\mathrm{I}\left({ }^{2} \mathrm{P}_{3 / 2}\right)$ ionization energy of $10.45 \mathrm{eV}$, it cannot be used to detect both I atom spin-orbit state channels with equal probability, as the $200-\mathrm{eV}$ electron impact spectrum can. The same $P\left(E_{\mathrm{T}}\right)$ does not fit both sets of spectra. Clearly, there is a difference in the ionization cross section at $10.8 \mathrm{eV}$ for the two different iodine states, leading to the preferential ionization of one of 


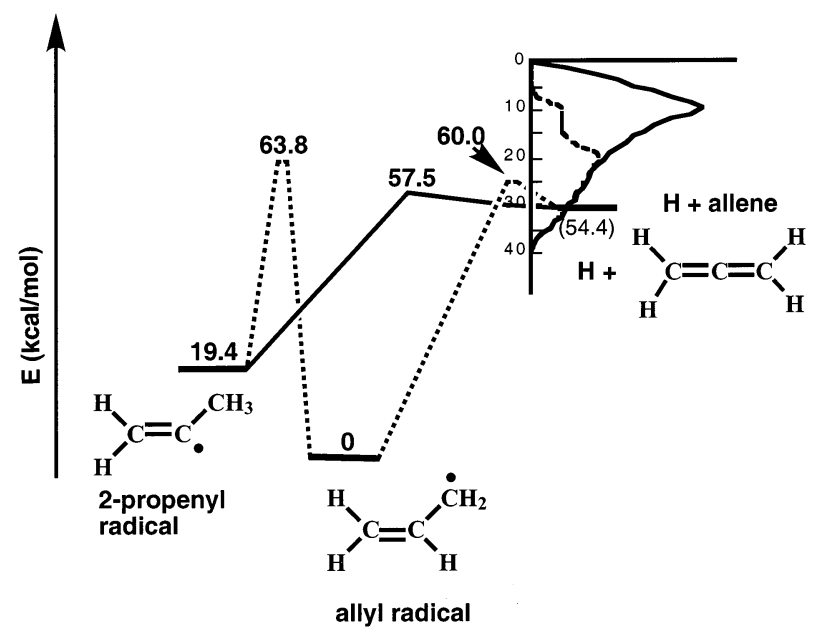

Figure 15. Schematic of the ab initio barrier energetics calculated in ref 23 for the allyl radical's $\mathrm{C}-\mathrm{H}$ bond fission channel and the isomerization channel to 2-propenyl. The inset is superimposed on the energy-level diagram to illustrate the distribution of internal energies in the nascent allyl radicals from the $\mathrm{I}\left({ }^{2} \mathrm{P}_{1 / 2}\right)$ channel. (The calculated barrier of $56.5 \mathrm{kcal} / \mathrm{mol}$ for the dissociation of the 2-propenyl radical to propyne $+\mathrm{H}$ is not shown.) The $P\left(E_{\mathrm{T}}\right)$ shown in the inset uses a vertical $P\left(E_{\mathrm{T}}\right)$ axis, but the vertical placement of the inset indicates the internal-energy spread of the allyl radical. The dashed-line $P\left(E_{\mathrm{T}}\right)$ displays the internal energy of the allyl radicals that are stable to dissociation and are therefore detected at $m / e=41$. As can be seen, because of centrifugal effects, the dashed line extends well over the barrier height of $60 \mathrm{kcal} / \mathrm{mol}$ (corresponding to $24 \mathrm{kcal} / \mathrm{mol}$ in translational energy), signaling the presence of allyl radicals that are stable to dissociation.

the states. Indeed, we expect that to allow indiscriminate detection of I atoms in both spin-orbit states, one would need to use a photoionization energy of $13.25 \mathrm{eV}$ to access the region of the I atom photoionization spectrum above the autoionization structure. ${ }^{37}$ Because I atoms in either the ${ }^{2} \mathrm{P}_{3 / 2}$ or the ${ }^{2} \mathrm{P}_{1 / 2}$ state share the same ionization cross section when undergoing highenergy impact ionization, we use the $P\left(E_{\mathrm{T}}\right)$ derived from that spectrum to match the momentum of the $m / e=40$ fragments produced from the secondary dissociation of the allyl radical accurately, as shown in Figure 7. This supports the conclusion that the $\mathrm{I}\left({ }^{2} \mathrm{P}_{1 / 2}\right)$ channel is a minor one because we did not have to subtract the $m / e=41 P\left(E_{\mathrm{T}}\right)$ from the $m / e=127 P\left(E_{\mathrm{T}}\right)$ to obtain a good fit.

As explained in the previous section, there is clearly some $m / e=41$ signal that remains from the $\mathrm{C}-\mathrm{I}$ bond fission event that should be unstable because the event partitioned less than $23.6 \mathrm{kcal} / \mathrm{mol}$ to product translation, leaving the allyl radical with more than $60 \mathrm{kcal} / \mathrm{mol}$ of internal energy. Figure 15 shows the barrier heights for the allene $+\mathrm{H}$ channel and the 2-propenyl isomerization channel along with a plot of the allyl radicals' internal energies. The presence of allyl radicals that do not dissociate despite having internal energies greater than $60 \mathrm{kcal} /$ mol could be reconciled if the barrier to dissociation of the allyl radical were more than $15 \mathrm{kcal} / \mathrm{mol}$ higher than the literature value, but this is unlikely because there are two independent calculations in the literature that place the barrier between 59 and $63 \mathrm{kcal} / \mathrm{mol} .{ }^{4,7}$ Clearly, we are observing allyl radicals that are stable to secondary dissociation even though they have internal energies well above the barrier height.

The origin of the stability to dissociation of a portion of the allyl radicals with an excess of up to $15 \mathrm{kcal} / \mathrm{mol}$ above the $\mathrm{C}-\mathrm{H}$ bond fission barrier height undoubtedly lies in centrifugal effects. Other possibilities are easily dismissed. The first excited state of the allyl radical, $\tilde{\mathrm{A}}\left(1^{2} \mathrm{~B}_{1}\right)$, lies $70.8 \mathrm{kcal} / \mathrm{mol}$ above the

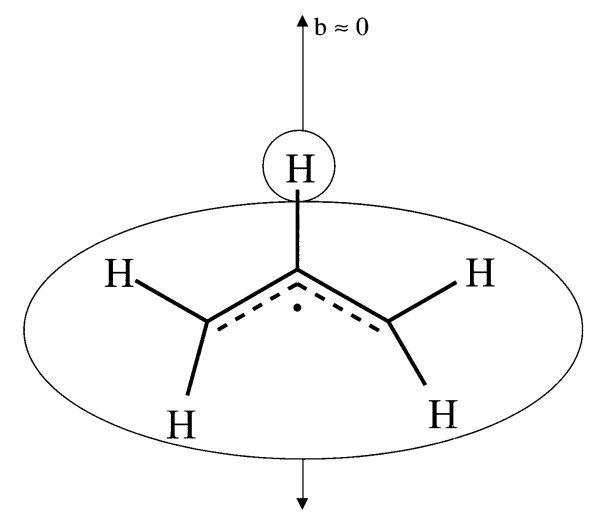

Figure 16. Picture displaying the near-zero impact parameter, $b$, characterizing the loss of an $\mathrm{H}$ atom to produce allene $+\mathrm{H}$. This nearzero impact parameter and the small reduced mass of the system are responsible for the marked centrifugal effects in the dissociation of rotationally excited allyl radicals seen here.

ground state, ${ }^{38}$ but this state is known to dissociate via conversion to the cyclopropyl radical ground state followed by ring opening to form the allyl radical $\tilde{X}$ state. We thus attribute the stability of the high internal energy allyl radical produced from the $\mathrm{I}\left({ }^{2} \mathrm{P}_{1 / 2}\right)$ channel to high rotational energy imparted to the allyl radical fragment during photolysis. A rigid radical impulsive model ${ }^{39}$ predicts that $41 \%$ of the available energy should be partitioned to rotational energy. Whereas the photodissociation of allyl iodide clearly partitions less energy to rotation than does the impulsive rigid radical limit, the high translational recoil imparted in the primary photolysis step and the long lever arm to the $\mathrm{C}_{3} \mathrm{H}_{5}$ center of mass likely result in considerable partitioning of energy to product rotation. The allyl radical simply cannot convert much of this rotational energy to angular momentum in the $\mathrm{H}+$ allene product recoil, $\mu\left|v_{\text {rel }}\right| b$, because allyl radical $\mathrm{C}-\mathrm{H}$ bond fission is characterized by a small impact parameter, $b$ (see Figure 16), and a small reduced mass, $\mu$. Much of the radicals' rotational energy must appear in the rotational energy of the allene product to conserve total angular momentum. Thus, the unimolecular dissociation of the rotationally excited allyl radical is expected to require much higher internal energy than one might predict using the ab initio barrier. Whereas detailed modeling of the centrifugal effects requires first modeling the energy imparted to the rotational energy of the allyl radical in the primary photolysis step, it is clear that our experimental results are consistent with this picture. Not only do we see stable allyl radicals with internal energy well above the $60 \mathrm{kcal} / \mathrm{mol}$ barrier, but the radicals that do dissociate yield surprisingly high-velocity $\mathrm{H}$ atoms with total recoil kinetic energies as high as $28 \mathrm{kcal} / \mathrm{mol}$. This suggests that the allyl radicals that do dissociate are, by virtue of their high rotational energy, experiencing significant centrifugal effects that shift the distribution of $\left|v_{\text {rel }}\right|$ to higher velocities. The conservation of angular momentum requires that the angular momentum imparted to the allyl radical fragment be composed of $\mathrm{C}-\mathrm{H}$ fission recoil angular momentum, $\mu\left|v_{\text {rel }}\right| b$, and allene angular momentum. Because of the near-zero impact parameter, $b$, and small reduced mass, $\mu,\left|v_{\text {rel }}\right|$ must increase to form rotationally colder allene products. Furthermore, the rotationally excited high-energy allyl radicals cannot isomerize to the 2-propenyl radical and dissociate with a larger impact parameter because the effective isomerization barrier is higher for rotationally excited allyl radicals.

It is interesting that centrifugal effects do not appear to be significant in the $\mathrm{I}\left({ }^{2} \mathrm{P}_{3 / 2}\right)$ formation channel. The $m / e=40$ spectrum (Figure 6) was accurately fit using the $P\left(E_{\mathrm{T}}\right)$ describing 
TABLE 1: Ratio of the RRKM Rate Constants for the Allyl $\rightarrow$ 2-Propenyl Isomerization Channel and the Direct Allyl $\rightarrow$ $\mathbf{H}+$ Allene Bond Fission Channel Calculated for Both a Low and a Higher Isomerization Barrier ${ }^{a}$

\begin{tabular}{ccc}
\hline & \multicolumn{2}{c}{$k_{\text {allyl } \rightarrow 2-\text { propenyl isom }} / k_{\text {allyl } \rightarrow \mathrm{H}+\text { allene }}$} \\
\cline { 2 - 3 }$E^{*}(\mathrm{kcal} / \mathrm{mol})$ & $48.6 \mathrm{kcal} / \mathrm{mol} \mathrm{barrier}^{b}$ & $63.8 \mathrm{kcal} / \mathrm{mol}$ barrier $^{c}$ \\
\hline 60.0 & $17.3 \times 10^{3}$ & \\
64.0 & 340 & $7.88 \times 10^{-3}$ \\
68.0 & 97.1 & $3.28 \times 10^{-2}$ \\
72.0 & 40.2 & $4.86 \times 10^{-2}$ \\
74.0 & 28.7 & $5.47 \times 10^{-2}$ \\
76.0 & 21.2 & $5.94 \times 10^{-2}$ \\
78.0 & 18.2 & $6.43 \times 10^{-2}$
\end{tabular}

${ }^{a}$ The calculations used the ab initio RRKM parameters (frequencies at the transition state and moments of inertia) reported in ref 23 . The rate constants for the allyl $\rightarrow 2$-propenyl isomerization were calculated using the most commonly cited experimental determination ${ }^{24}$ of the barrier of $48.6 \mathrm{kcal} / \mathrm{mol}$ as well as the ab initio barrier ${ }^{23}$ of $63.8 \mathrm{kcal} /$ mol. As can be seen, when the lower barrier is assumed, it predicts that the rate of isomerization dominates the direct allyl radical $\rightarrow \mathrm{H}+$ allene formation, whereas direct $\mathrm{H}+$ allene formation is favored when the $63.8 \mathrm{kcal} / \mathrm{mol}$ allyl $\rightarrow$ 2-propenyl isomerization barrier is assumed. Particularly at near-threshold internal energies, where the 2-propenyl radical has a fast $\mathrm{H}+$ propyne dissociation channel, the faster isomerization to 2-propenyl calculated by assuming that the lower barrier is correct would give a dominant branching to the $\mathrm{H}+$ propyne product, in contradiction to our experimental results. ${ }^{b}$ From ref 24. ${ }^{c}$ From ref 23.

$\mathrm{C}-\mathrm{I}$ bond fission (Figure 2) without truncation. One possible reason for this lack of centrifugal effects in the $\mathrm{I}\left({ }^{2} \mathrm{P}_{3 / 2}\right)$ channel is the preferential production of rotationally hot radicals in the $\mathrm{I}\left({ }^{2} \mathrm{P}_{1 / 2}\right)$ channel. Prior work by Hintsa et al. ${ }^{34}$ also attributed the stability of some of the $\mathrm{C}_{2} \mathrm{H}_{4} \mathrm{OH}$ photofragments in 2-bromoethanol photodissociation to metastability from the rotational energy imparted to $\mathrm{C}_{2} \mathrm{H}_{4} \mathrm{OH}$ in the primary $\mathrm{C}-\mathrm{Br}$ fission.

The other important result from this work is the clear evidence that the previous experimental determination of the barrier height for allyl $\rightarrow$ 2-propenyl isomerization is too low. Whereas the two recent ab initio studies predict the barrier to be near 64 $\mathrm{kcal} / \mathrm{mol},{ }^{4,7}$ the 1986 experimental study determined the barrier to be $48.6 \mathrm{kcal} / \mathrm{mol}^{24}$ RRKM calculations were performed ${ }^{40}$ using the barrier heights and frequencies of Davis et al. as well as the $48.6-\mathrm{kcal} / \mathrm{mol}$ barrier height to determine the expected major pathways at the energies available here. The centrifugal barrier was not accounted for because we are interested in the relative rates, and the effect of the centrifugal barrier should be similar for the allene $+\mathrm{H}$ channel and the 2-propenyl isomerization channel. Results are shown in Table 1 . When the lower barrier to 2-propenyl isomerization of $48.6 \mathrm{kcal} / \mathrm{mol}$ is assumed, the rate of allyl $\rightarrow$ 2-propenyl isomerization dominates the direct dissociation of allyl to form allene $+\mathrm{H}$ at the energies available here. The allene/propyne formation branching ratio of the 2-propenyl radical is known to be dependent on internal energy. ${ }^{41}$ Propyne formation is favored at energies just above the allene $+\mathrm{H}$ barrier. Experimental results on the 2-propenyl radical have shown that only $21 \%$ of 2 -propenyl radicals with median internal energy that is $15 \mathrm{kcal} / \mathrm{mol}$ over the $\mathrm{H}+$ allene barrier actually produced allene $+\mathrm{H}^{41}$ Thus, if the lower barrier to 2-propenyl isomerization of $48.6 \mathrm{kcal} / \mathrm{mol}$ is assumed, one would expect to see a significant propyne contribution at nearthreshold internal energies. Even though the $m / e=40$ PIE curve (Figure 7) cannot entirely rule out propyne formation, the large propyne contribution expected because of a low 2-propenyl isomerization barrier is clearly absent, as is shown in Figure 8. In that data, if the isomerization barrier were low, the rate constant for isomerization to the 2-propenyl radical would be over 100 times larger than the rate constant of the direct $\mathrm{H}+$ allene channel for the allyl radical at internal energies of 64 $\mathrm{kcal} / \mathrm{mol}$ in the allyl radical. Then, the fast edge of the $m / e=$ 40 TOF spectra would have been dominated by propyne, and the 10.0-eV spectra, below the propyne ionization energy, would have been markedly reduced from the fast edge on the $10.8-\mathrm{eV}$ spectrum. Clearly, the fast edge is not reduced in the $10.0-\mathrm{eV}$ spectrum. Thus, undoubtedly, the allene product channel dominates at these near-threshold internal energies. Only the higher isomerization barrier predicted in the theoretical calculations is consistent with our experimental results; the experimentally determined barrier is far too low.

Note Added in Proof. The work in refs 3 and 7 has been recently reviewed in Fischer, I.; Chen, P. J. Phys. Chem. A 2002, 106, 4291.

Acknowledgment. This work was supported by the Division of Chemical Sciences, Office of Basic Energy Sciences, Office of Energy Research, U.S. Department of Energy, under Grant No. DE-FG02-92ER14305 (L. J. B.). The Chemical Dynamics Beamline is supported by the Director, Office of Science, Office of Basic Energy Sciences, Chemical Sciences Division of the U. S. Department of Energy under contract No. DE-AC0376SF00098. The ALS facility is supported by the Director, Office of Science, Office of Basic Energy Sciences, Materials Sciences Division of the U.S. Department of Energy, under the same contract. The authors would like to acknowledge Fei Qi for his contribution and data collection at the start of the project, Yi Liu for her help with the HRTOF study, and Maria Krisch for her help in the $\mathrm{H}$ atom partner fragment data acquisition. The construction costs of the HRTOF apparatus were supported in part by a CRIF grant, NSF CHE-9974937.

\section{References and Notes} 2025 .

(1) Minsek, D. W.; Blush, J. A.; Chen, P. J. Phys. Chem. 1992, 96 ,

(2) Minsek, D. W.; Chen, P. J. Phys. Chem. 1993, 97, 13375.

(3) Deyerl, H.-J.; Gilbert, T.; Fischer, I.; Chen, P. J. Chem. Phys. 1997 107,3329

(4) Stranges, D.; Stemmler, M.; Yang, X.; Chesko, J. D.; Suits, A. G.; Lee, Y. T. J. Chem. Phys. 1998, 109, 5372.

(5) Schultz, T.; Clarke, J. S.; Gilbert, T.; Deyerl, H.-J.; Fischer, I. Faraday Discuss. 2000, 115, 17.

(6) Wu, J.-C.; Li, R.; Chang, J.-L.; Chen, Y.-T. J. Chem. Phys. 2000, 113,7286

(7) (a) Deyerl, H.-J.; Fischer, I.; Chen, P. J. Chem. Phys. 1999, 110, 1450. (b) Deyerl, H.-J. Ph.D. Thesis, ETH Zürich, Zürich, Switzerland, 1999.

(8) Nandi, S.; Arnold, P. A.; Carpenter, B. K.; Nimlos, M. R.; Dayton, D. C.; Ellison, G. B. J. Phys. Chem. A 2001, 105, 7514

(9) Jenkin, M. E.; Murrells, T. P.; Shalliker, S. J.; Hayman, G. D. J. Chem. Soc., Faraday Trans. 1993, 89, 433.

(10) Getty, J. D.; Burmeister, M. J.; Westre, S. G.; Kelly, P. B. J. Am. Chem. Soc. 1991, 113, 801

(11) Getty, J. D.; Liu, X.; Kelly, P. B. J. Phys. Chem. 1992, 96, 10155

(12) Worrell, C. W. J. Electron Spectrosc. Relat. Phenom. 1974, 3, 359.

(13) Boschi, R. A.; Salahub, D. R. Mol. Phys. 1972, 24, 735.

(14) Parsons, B. F.; Szpunar, D. E.; Butler, L. J. J. Phys. Chem. A 2000, 104, 10669.

(15) Baklanov, A. V.; Maltsev, V. P.; Karlsson, L.; Lindgren, B.; Sassenberg, U. Chem. Phys. 1994, 184, 357.

(16) Baklanov, A. V.; Aldener, M.; Lindgren, B.; Sassenberg, U. J. Chem. Phys. 2000, 112, 6649.

(17) Arnold, P.; Cosofret, B.; Dylewski, S.; Houston, P.; Carpenter, B. Private communication of results on allyl iodide at $266 \mathrm{~nm}$.

(18) Baklanov, A. V.; Maltsev, V. P.; Karlsson, L.; Sassenberg, U.; Persson, A. J. Chem. Soc., Faraday Trans. 1996, 92, 1681. 
(19) Barnes, A. J.; Holroyd, S. Spectrochim. Acta, Part A 1983, 39, 579. (20) Camaggi, C. M.; Leardini, R.; Zanirato, P. J. Org. Chem. 1977, 42,1570 .

(21) All $\Delta H^{\circ}$ values were taken from NIST Chemistry WebBook, NIST Standard Database Number 69; National Institute of Standards and Technology: Gaithersburg, MD 20899 (July 2001) except for $\Delta H^{\circ}$ (allene), which was taken from ref 22 .

(22) CRC Handbook of Chemistry and Physics, 82nd ed.; Lide, D. R., Ed.; CRC Press: Boca Raton, FL, 2001-2002.

(23) Davis, S. G.; Law, C. K.; Wang, H. J. Phys. Chem. A 1999, 103 5889.

(24) Ondruschka, V. H.; Ziegler, U.; Zimmerman, G. Z. Phys. Chem. (Leipzig) 1986, 267, 1127.

(25) Niedzielski, J.; Gawlowski, J.; Gierczak, T. J. Photochem. 1983, $21,195$.

(26) Person, M. D. Ph.D. Thesis, University of Chicago, Chicago, IL, 1991.

(27) Daly, N. R. Rev. Sci. Instrum. 1960, 31, 264.

(28) Yang, X.; Lin, J.; Lee, Y. T.; Blank, D. A.; Suits, A. G.; Wodtke, A. M. Rev. Sci. Instrum. 1997, 68, 3317.

(29) Proch, D.; Trickl, T. Rev. Sci. Instrum. 1989, 60, 713.
(30) Schnieder, L.; Meier, W.; Welge, K. H.; Ashfold, M. N. R.; Western, C. M. J. Chem. Phys. 1990, 92, 7027.

(31) Parsons, B. F. Ph.D. Thesis, University of Chicago, Chicago, IL, 2001.

(32) Values taken from ref 21

(33) Baer, T.; Hase, W. L. Unimolecular Reaction Dynamics: Theory and Experiments; Oxford University Press: New York, 1996.

(34) Hintsa, E. J.; Zhao, X.; Lee, Y. T. J. Chem. Phys. 1990, 92, 2280.

(35) Morton, M. L.; Butler, L. J.; Stephenson, T. A.; Qi, F. J. Chem. Phys. 2002, 116, 2763.

(36) Morton, M. L.; Miller, J. L.; Butler, L. J.; Qi, F. J. Phys. Chem. A, in press.

(37) Berkowitz, J.; Batson, C. H.; Goodman, G. L. Phys. Rev. A 1981, 24,149 .

(38) Currie, C. L.; Ramsay, D. A. J. Chem. Phys. 1966, 45, 488.

(39) Riley, S. J.; Wilson, K. R. Faraday Discuss. Chem. Soc. 1972, 53, 132.

(40) Hase, W. L.; Bunker, D. L. QCPE 1974, 234 (A General RRKM Program).

(41) Mueller, J. A.; Parsons, B. F.; Butler, L. J.; Qi, F.; Sorkhabi, O.; Suits, A. G. J. Chem. Phys. 2001, 114, 4505. 